\title{
Theory of freestanding triboelectric-layer-based nanogenerators
}

Simiao Niu ${ }^{a}$, Ying Liu ${ }^{a}$, Xiangyu Chen ${ }^{b}$, Sihong Wang ${ }^{a}$, Yu Sheng Zhou ${ }^{a}$, Long Lin ${ }^{a}$, Yannan $X_{i e}{ }^{a}$, and Zhong Lin Wang ${ }^{a, b, *}$

${ }^{a}$ School of Materials Science and Engineering, Georgia Institute of Technology, Atlanta, Georgia 30332-0245, United States

${ }^{\mathrm{b}}$ Beijing Institute of Nanoenergy and Nanosystems, Chinese Academy of Sciences, Beijing, China

*Corresponding author at: School of Materials Science and Engineering, Georgia Institute of Technology, Atlanta, Georgia, 30332-0245, United States

E-mail address: zlwang@gatech.edu (Z. L. Wang)

\begin{abstract}
:
Triboelectric nanogenerator technology is emerging as a promising candidate for mechanical energy harvesting from ambient environment. Freestanding triboelectric-layer-based nanogenerators (FTENGs) are one of the fundamental operation modes with many advantages. In this paper, the first theoretical model of FTENGs is proposed with thorough analysis of their operation principle. Both contact-mode and sliding-mode FTENGs are discussed to fully uncover their unique characteristics. Contact-mode FTENGs have superior linearity, which is highly beneficial for both energy-harvesting and self-powered sensing applications. Slidingmode FTENGs have two subcategories based on the material of their freestanding layer. For both of the two subcategories, the coupling effect of the height of the freestanding layer and the electrode gap on their output characteristics are discussed in detail to obtain the strategy for their structural optimization.
\end{abstract}

Keywords: mechanical energy harvesting, freestanding triboelectric nanogenerator, structural optimization

\section{Introduction}


Energy harvesting from natural environment has long been considered as a promising supplement to the traditional fuel sources. It can not only contribute to the hugely increased electricity demand of modern society, but also solve the energy source problem for mobile electronics when the traditional energy source is unreachable. Among all of the energy sources, mechanical energy has attracted much attention, mainly for its wide availability and high operability. Effects such as the electromagnetic,[1,2] electrostatic,[3-6] and piezoelectric effect[7] have already been utilized in mechanical energy harvesting, but each of them has its own limitations. Heavy magnets and pre-charging process are indispensable for electromagnetic generators and electrostatic electret generators, respectively. In addition, the low output of piezoelectric generators still limits their applicability. Recently, triboelectric nanogenerators (TENGs) based on the coupling effect of contact electrification[8-11] and electrostatic induction have been invented to overcome the above limitations and have exhibited their unique merits, such as large output power, high efficiency, cost effective materials, low weight, and simple fabrication.[12] Several operation modes of TENGs have already been developed to adapt them to different applications.[12-17] Among all of the operation modes, freestanding triboelectric-layer-based nanogenerators (FTENGs) have the following unique advantages.[16-19] First, compared to traditional attached-electrode TENGs, the moving triboelectric layer in FTENGs is not necessary to be attached with an electrode and a lead wire, which makes this device applicable to harvest mechanical energy from any arbitrary moving objects, such as a walking human and a moving automobile.[16] Second, compared to single-electrode TENGs with similar advantage discussed above,[20] FTENGs effectively avoid the electrostatic shield effect, so the limit of the charge transfer efficiency of FTENGs can be close to $100 \%$, much larger than that of single-electrode TENGs $(50 \%)$. Finally, FTENGs can work in the non-contact mode without significant degradation of the output, which can tremendously increase the energy conversion efficiency due to the large reduction of energy loss from both friction and inelastic collision in the system._ENREF_16 
However, until now fundamental understandings of FTENGs are still missing to reveal unique characteristics of their output performance. First, their operation principle and the fundamental physics need to be uncovered. Second, their unique output characteristics need to be thoroughly studied to assist the rational design of this structure. Finally, the influence of the structural parameters and how to optimize them to obtain the highest performance is unclear yet. Therefore, a comprehensive theoretical analysis of this structure is necessary.

In this paper, the first theoretical models of two types of FTENGs: contact-mode FTENGs based on vertical charge separation and sliding-mode FTENGs based on in-plane charge separation are discussed in detail. Contact-mode FTENGs are observed to have superior linear characteristics, which give rise to unique applications in vibration energy-harvesting and sensing. Sliding-mode FTENGs have two subcategories based on the material of the freestanding layer. Their output characteristics are carefully studied and the coupling effect of two important design parameters, freestanding height and electrode gap, on the output characteristics of these two sub-categories is investigated. In addition, structural optimization strategies are provided to maximize the power output for both contact-mode and sliding-mode FTENGs. This paper thoroughly elucidates the core working principle and unique characteristics of FTENGs and systematically investigates the influence of different structural parameters, which can serve as an important guideline and pave the way for their rational design and optimization towards real applications.

\section{Contact-mode freestanding triboelectric-layer-based nanogenerators}

\subsection{Theoretical model of the contact-mode freestanding triboelectric-layer-based nanogenerator}

We start our discussion from the contact-mode freestanding triboelectric-layer-based nanogenerators (CFTENGs). Two typical structures of CFTENGs are shown in Figure 1. Figure 1a shows a typical structure for dielectric-freestanding-layer CFTENGs. A dielectric 
plate (thickness: $d_{1}$, relative dielectric constant: $\varepsilon_{\mathrm{r} 1}$ ) and two metal plates are stacked face to face, forming two triboelectric pairs. The two metal plates also serve as two electrodes. The total air gap thickness between these two metal plates is defined as $g$. After the dielectric plate being forced to contact with the two metal plates, both the top and the bottom surfaces of Dielectric 1 will have static triboelectric charges (tribo-charge) due to contact electrification. For simplicity, we assume the triboelectric charge density of both surfaces is the same $(-\sigma)$. At the same time, the two metal plates will have the same amount of positive charges in total because of charge conservation.

The concept of nodes is utilized to analyze this electrostatic system, which can fully show its inherent physics and working behavior.[20] In practical applications, the size of the metal electrode is always much larger than the air gap. Therefore, the area size of the freestanding contact-mode TENG $(S)$ is seen as infinitely large and the edge effect can be ignored. As a result, the electric potential on metal 1 , metal 2 , the top and bottom surface of dielectric 1 are all constant, so these 4 surfaces can be seen as 4 nodes in this electrostatic system. (A node is defined as a surface/volume with a certain electrical potential) The electrical line connection between every two nodes forms an equivalent capacitance between them. Since the area size $(S)$ is assumed as infinitely large, the electrical line connection between every two nonadjacent nodes (for example, Node $1 \& 3$ ) is fully blocked by the intermediate node (for example, Node 2). Therefore, only three capacitances exist in the equivalent circuit model of this electrostatic system, as shown in Figure 1c. The total capacitance between the two electrodes (Node $1 \& 4$ ) is the serial connection of $C_{1}, C_{2}$, and $C_{3}$, which can be easily given by the following equation.

$$
C=\frac{1}{\frac{1}{C_{1}}+\frac{1}{C_{2}}+\frac{1}{C_{3}}}=\frac{\varepsilon_{0} S}{d_{0}+g}
$$

Inside equation 1 , the effective dielectric thickness $d_{0}$ is defined as the summation of all the thickness of the dielectric $d_{i}$ inside the air gap divided by its relative effective thickness $\varepsilon_{r i}$, as 
shown below.

$$
d_{0}=\sum_{i=1}^{n} \frac{d_{i}}{\varepsilon_{r i}}
$$

The charge distribution under short circuit (SC) condition when Node 1 and Node 4 have the same electrical potential is then analyzed. The charges on each node need to be deducted to solve this electrostatic system. For both Node 2 and 3, the amount of charges on the node is $\sigma S$. In addition, because of charge conservation, we only know that the total charges on Node 1 and 4 are $2 \sigma S$, but the detailed charge distribution is still unknown. Under SC condition, we set the total charges on metal 1 (Node 1) is $Q_{1}$ and the total charges on metal 2 (Node 4 ) is $Q_{2}$ $\left(Q_{2}=2 \sigma S-Q_{1}\right)$. From the basic electrodynamics theory and charge conservation, the following equation set can be obtained.

$$
\begin{gathered}
V_{1}=\frac{Q_{1}}{C_{1}}(3 a) \\
V_{2}=-\frac{\sigma S-Q_{1}}{C_{2}}(3 b) \\
V_{3}=-\frac{2 \sigma S-Q_{1}}{C_{3}}(3 c) \\
V_{1}+V_{2}+V_{3}=0 \quad(3 d)
\end{gathered}
$$

From the above equation set, the total charges on metal 1 and 2 can be easily solved as:

$$
Q_{1}=\sigma S \frac{\frac{1}{C_{2}}+\frac{2}{C_{3}}}{\frac{1}{C_{1}}+\frac{1}{C_{2}}+\frac{1}{C_{3}}}(4 a) \quad Q_{2}=\sigma S \frac{\frac{2}{C_{1}}+\frac{1}{C_{2}}}{\frac{1}{C_{1}}+\frac{1}{C_{2}}+\frac{1}{C_{3}}}
$$

In practical applications, the effective dielectric thickness of the dielectrics $d_{1} / \varepsilon_{\mathrm{r} 1}$ is always negligible compared to the air gap and $C_{2}$ can be seen as infinitely large. Thus, equation (4a) and (4b) can be further simplified to:

$$
Q_{1} \approx \sigma S \frac{\frac{2}{C_{3}}}{\frac{1}{C_{1}}+\frac{1}{C_{3}}}=\frac{2 \sigma S}{1+\frac{C_{3}}{C_{1}}}(5 a) \quad Q_{2} \approx \frac{2 \sigma S}{1+\frac{C_{1}}{C_{3}}}(5 b)
$$

From equation $5 \mathrm{a}$ and $5 \mathrm{~b}$, the basic working mechanism of CFTENGs can be easily observed. 
When $x=0, C_{3}$ is infinity, as a result, $Q_{1}$ is close to 0 and $Q_{2}$ is approximately $2 \sigma S$. Under this condition, all the positive tribo-charges are attracted to the bottom electrode by the negative charges on the surface of dielectric 1 . While when $x=g, C_{1}$ is infinity and all the positive charges on the electrodes are attracted to Metal 1 . Therefore, if Dielectric 1 is under a vibration inside the air gap, charges will alternatively flow between Metal 1 and Metal 2 due to the change of $C_{1} / C_{3}$, forming an $\mathrm{AC}$ short circuit current. Thus, the change of the capacitance ratio between the tribo-charged surfaces and the two electrodes induced by the change of Dielectric 1 position can drive electrons to flow under SC conditions between the two electrodes, which is the main working principle of CFTENGs.

To calculate the transferred charges $(Q)$, we need to pick up a charge reference state, which is the state when Metal 1 has a specific number of charges $\left(Q_{0}\right)$. Then the transferred charges $(Q)$ can be calculated by deducting $Q_{0}$ from the current charge amount on Metal 1 . The number of $Q_{0}$ can be arbitrarily picked, which will not affect any calculation results for voltage or current. (Detailed discussion of charge reference state is shown in ESI, Section 1.) The most commonly utilized charge reference state is called minimum achievable charge reference state (MACRS), in which $Q_{0}$ is assigned to the total amount of charges on metal 1 under SC condition when $x=0$, as shown in equation 6 .

$$
Q_{0}=Q_{1}(0)=\frac{\sigma S d_{0}}{d_{0}+g}
$$

Under MACRS, the short circuit transferred charges $\left(Q_{\mathrm{SC}}\right)$ can be calculated as:

$$
Q_{S C}=Q_{1}-Q_{1}(0)=\frac{2 \sigma S x}{d_{0}+g}
$$

From the basic equation between $V_{\mathrm{OC}}, Q_{\mathrm{SC}}$ and $C$, [20, 21] $V_{\mathrm{OC}}$ (under MACRS) can be easily obtained as equation 8 .

$$
V_{O C}=\frac{Q_{S C}}{C}=\frac{2 \sigma x}{\varepsilon_{0}}
$$

Therefore, the governing equation for this CFTENG, which is its $V-Q-x$ relationship, [21] can be shown as: 


$$
V=-\frac{1}{C} Q+V_{O C}=-\frac{d_{0}+g}{\varepsilon_{0} S} Q+\frac{2 \sigma x}{\varepsilon_{0}}
$$

Besides the dielectric-freestanding-layer CFTENGs, another important category is metalfreestanding-layer CFTENGs, as shown in Figure 1c. The working principle of metalfreestanding-layer CFTENGs is very similar to the dielectric-freestanding-layer case. Similar to the traditional contact-mode attached-electrode TENG,[22] metal can be simply seen as dielectrics with 0 thickness to calculate $d_{0}$ and the derived equations above are all applicable to metal CFTENGs.

From the above derivation, it can be easily observed that for contact-mode FTENGs, both $V_{\mathrm{OC}}$ and $Q_{\mathrm{SC}}$ has linear dependency with the separation distance $x$. This theoretical conclusion can also be verified by the experimental results, as shown in Figure 2 for a dielectricfreestanding-layer TENG case. (Detailed experiment procedure is shown in ESI, Section 6). The experimental measured $V_{\mathrm{OC}}$ and $Q_{\mathrm{SC}}$ both show accurate linear dependence with $x$ and the correlation coefficients for both curves are more than 0.99987. The demonstrated experimental results match with our theoretical anticipation very well, which again shows our models are accurate and our assumptions are valid.

With the above information, the comparison of the basic output characteristics of CFTENGs and contact-mode attached-electrode TENGs[22] can be provided. Their $V_{\mathrm{OC}}$ characteristics are similar to each other, which are both linearly proportional to $x$. However, their inherent capacitance and short-circuit transferred charges characteristics are completely different. First, the inherent capacitance of CFTENGs is constant and independent of $x$ while that of contactmode attached-electrode TENGs decreases when $x$ increases. In addition, $Q_{\mathrm{SC}}$ of FETENGs is also linearly proportional to $x$ while that of attached-electrode TENGs has a saturation trend.

\subsection{Resistive load output characteristics}

When a CFTENG is connected with a resistive load, the equivalent circuit model of the whole system is shown in Figure 3a.[23] This CFTENG system is completely linear and time- 
invariant because the inherent capacitance of CFTENGs is time-invariant and their $V_{\mathrm{OC}}$ is completely linear with $x$. To solve this linear time-invariant system, the external mechanical motion must be specified. As a typical example, the mechanical motion is chosen as a single frequency harmonic vibration (shown as Equation 10), so the open-circuit voltage source in the equivalent circuit model is also a single frequency harmonic signal, as shown below.

$$
\begin{gathered}
x=x_{0}+A_{0} \sin (\omega \mathrm{t}) \\
V_{O C}=\frac{2 \sigma x}{\varepsilon_{0}}=\frac{2 \sigma}{\varepsilon_{0}}\left(x_{0}+A_{0} \sin (\omega \mathrm{t})\right)
\end{gathered}
$$

With this single-frequency harmonic signal as the input of the linear time-invariant system, the steady-state output will also be a single frequency harmonic signal. Utilizing phase method, the steady-state voltage and current output of the resistive load can be easily derived as:

$$
\begin{gathered}
V=\frac{R}{\frac{1}{j \omega C}+R} \frac{2 \sigma A_{0}}{\varepsilon_{0}}=\frac{2 \sigma A_{0}}{\varepsilon_{0}} \frac{\omega R C}{1+\omega^{2} R^{2} C^{2}}(\omega R C+j) \quad(12 a) \\
V(t)=\frac{2 \sigma A_{0}}{\varepsilon_{0}} \frac{\omega R C}{1+\omega^{2} R^{2} C^{2}}(\omega R C \sin (\omega \mathrm{t})+\cos (\omega \mathrm{t}))=V_{0} \sin (\omega \mathrm{t}+\varphi) \\
I=\frac{V}{R}=\frac{2 \sigma A_{0}}{\varepsilon_{0}} \frac{\omega C}{1+\omega^{2} R^{2} C^{2}}(\omega R C+j) \\
I(t)=\frac{2 \sigma A_{0}}{\varepsilon_{0}} \frac{\omega C}{1+\omega^{2} R^{2} C^{2}}(\omega R C \sin (\omega \mathrm{t})+\cos (\omega \mathrm{t}))=I_{0} \sin (\omega \mathrm{t}+\varphi)
\end{gathered}
$$

The amplitude of the voltage and current output signal $\left(V_{0}\right.$ and $\left.I_{0}\right)$ and their phase $(\varphi)$ can be easily given by:

$$
\begin{aligned}
V_{0} & =\frac{2 \sigma A_{0} \omega R C}{\varepsilon_{0} \sqrt{1+\omega^{2} R^{2} C^{2}}}(14 a) \\
I_{0} & =\frac{2 \sigma A_{0} \omega C}{\varepsilon_{0} \sqrt{1+\omega^{2} R^{2} C^{2}}}(14 b) \\
\varphi & =\arctan \left(\frac{1}{\omega R C}\right) \quad(14 c)
\end{aligned}
$$

In addition, the effective power delivered to the load $\left(P_{\text {eff }}\right)$ is shown as:

$$
P_{\text {eff }}=\frac{V_{0}^{2}}{2 R}=\frac{\omega^{2} C^{2} R}{1+\omega^{2} R^{2} C^{2}} \frac{2 \sigma^{2} A_{0}^{2}}{\varepsilon_{0}^{2}}
$$

An optimum resistance to maximize the effective power can be easily observed from equation 
15, which is because of the impedance match between the TENG capacitance and the load resistance. The impedance match condition and the maximum effective power output can be derived easily from the derivative of equation 15, as shown in equation 16 and 17.

$$
R_{\mathrm{opt}}=\frac{1}{\omega C}=\frac{d_{0}+g}{\varepsilon_{0} S \omega}(16) \quad P_{\mathrm{eff}, \mathrm{opt}}=\frac{\sigma^{2} A_{0}^{2} \omega S}{\varepsilon_{0}\left(d_{0}+g\right)}
$$

To validate the above theoretical anticipation, a numerical calculation was done through the TENG-simulator[23] and the corresponding results are plotted in Figure 3b-d. (Detailed parameters utilized in this simulation are listed in Table I.) Consistent with the theoretical anticipation, all output curves are harmonic. When $R$ begins to increase from 0 to about 100 $\mathrm{M} \Omega$, the impedance of the TENG capacitance $(1 / j \omega C)$ is much larger than $R$. Therefore, the current profile stay close to that of SC profile and the phase shift of the output curve is approximately 90 degree. When $R$ continues to increase, the total impedance of the serial connection of $1 / j \omega C$ and $R$ starts to increase, resulting in a drop of the current signal amplitude and an increase of the voltage signal amplitude. At the same time, the phase shift of the output signal starts to decrease from 90 degree to 0 . When $R$ approaches infinity, the AC component of $V_{\mathrm{OC}}$ is totally applied to $R$. As a result, the voltage signal reaches its maximum amplitude and is in-phase with the mechanical motion.

\subsection{Superior linearity of contact-mode FTENGs}

The most advantage of this CFTENG structure is its superior linearity. The linear dependence of both $V_{\mathrm{OC}}$ and $Q_{\mathrm{SC}}$ on $x$ makes it an ideal medium to transform mechanical vibration signal to electrical signal. Besides, the constant inherent capacitance makes it a linear and timeinvariant device. Thus, the electrical signal output of this device is compatible with all the currently available signal processing techniques, making it easy to accomplish postprocessing of the obtained electrical signal. Therefore, this structure could have wide applications in self-powered vibration detection. 
Besides the self-powered sensor application, this superior linearity is also beneficial in vibration energy harvesting applications as well. To fully uncover its advantages, we design a device structure that can harvest vibration energy under both freestanding-mode and traditional attached-electrode mode, as shown in Figure 4a. When metal 1 and metal 2 are directly utilized as two electrodes and metal 3 and 4 are suspended with no output terminal, this device is working in the freestanding-mode (shown in the right solid line). If metal 1 and metal 4 are connected to form Node A, metal 2 and metal 3 are connected to form Node B and then Node A and Node B are utilized as the output terminals to connect with external load circuits, the device is working in traditional attached-electrode mode, which is equivalent to two parallel-connected attached-electrode TENGs. The reason to connect metal 1 and 4 rather than metal 1 and 2 to form the output node is to ensure the polarity of these two TENGs is consistent. For simplicity, we still assume the tribo-charge surface density on the surface of Dielectric 1 and 2 are the same value $(-\sigma) . V_{\mathrm{OC}}$ and $Q_{\mathrm{SC}}$ for the freestanding mode have already been shown as equation 7 and 9 while those for the attached-electrode mode $\left(V_{\mathrm{OC}, \mathrm{AE}}\right.$ and $\left.Q_{\mathrm{SC}, \mathrm{AE}}\right)$ are shown in the following equations. (The explanation of the parameters is shown in Table II. For a detailed derivation, see ESI, Section 2.)

$$
\begin{gathered}
V_{O C, A E}=\frac{\sigma x}{\varepsilon_{0}} \frac{\frac{\frac{d_{1}}{\varepsilon_{r 1}}}{\left(g+\frac{d_{1}}{\varepsilon_{r 1}}\right)\left(g+\frac{d_{1}}{\varepsilon_{r 1}}-x\right)}+\frac{1}{x+\frac{d_{2}}{\varepsilon_{r 2}}}}{\frac{1}{\frac{d_{3}}{\varepsilon_{r 3}}+\frac{1}{g+\frac{d_{1}}{\varepsilon_{r 1}}-x}+\frac{1}{x+\frac{d_{2}}{\varepsilon_{r 2}}}}} \\
Q_{S C, A E}=\sigma x S\left[\frac{\frac{d_{1}}{\varepsilon_{r 1}}}{\left(g+\frac{d_{1}}{\varepsilon_{r 1}}\right)\left(g+\frac{d_{1}}{\varepsilon_{r 1}}-x\right)}+\frac{1}{x+\frac{d_{2}}{\varepsilon_{r 2}}}\right]
\end{gathered}
$$

As a comparison, $Q_{\mathrm{SC}}$ and $V_{\mathrm{OC}}$ under both two operation-modes are numerically calculated utilizing the same parameters and plotted in Figure 4c and Figure S4, respectively. Although for both of these two-modes, their $Q_{\text {sc-final }}$ (short-circuit transferred charge amount when a full 
separation is reached) can reach $2 \sigma S$, the shape of $Q_{\mathrm{sc}}$ is completely different. For the freestanding mode, $Q_{\mathrm{sc}}$ changes linearly with $x$, ensuring a relative high slope in the whole $x$ range. However, for the traditional attached-electrode mode, $Q_{\mathrm{sc}}$ only has a huge slope at the two ends. When $x$ is between $0.05 g$ to $0.95 g, Q_{\text {sc }}$ is nearly a constant. Therefore, when the traditional attached-electrode mode is utilized to harvest vibration energy with vibration amplitude which is not large enough to ensure the freestanding layer to fully contact with the two electrodes (which is always the case in practical application), the total short-circuit transferred charges will be strongly limited. Besides $Q_{\mathrm{SC}}, V_{\mathrm{OC}}$ of the attached-electrode mode is also limited by the large capacitance generated between Metal 3 and 4 (small $d_{3}$ in equation 19), which will also result in a limited total energy harvested, as shown in Figure S4. As an example, this device is utilized to harvest a harmonic vibration whose vibration amplitude is $g / 4$ and center position is $x=g / 2$, as shown in Equation 20. The steady state electrical outputs under these two modes are numerically calculated through the TENG simulator utilizing the periodic boundary condition. $[23,24]$ As shown in Figure 4c, the amount of transferredcharges of the freestanding mode is more than 70 times higher than the attached-electrode mode in all the resistance ranges. In addition, the optimum energy harvested from the freestanding-mode is several orders larger than the attached-electrode mode. From the above comparison, we can conclude that the linearity of the freestanding mode is highly beneficial for harvesting the mechanical energy from the vibration that cannot ensure good contact of the moving object and the two electrodes, which will also save energy loss from the inelastic collision and increase the energy conversion efficiency. Theoretically, working in this noncontact mode, the CFTENG can have energy conversion efficiency as high as $100 \%$.

$$
x=\frac{g}{2}+\frac{g}{4} \sin (\omega \mathrm{t})
$$

\section{Sliding-mode freestanding triboelectric-layer-based nanogenerators}

\subsection{Basic properties and influence of the freestanding material}


Besides CFTENGs, another important category is the sliding-mode FTENGs (SFTENGs) based on in-plane charge separation mechanism. $[16,17]$ Based on the freestanding material, SFTENGs have two subcategories, dielectric SFTENGs whose freestanding materials only contain dielectrics and metal SFTENGs whose freestanding materials contains metal. The basic model of these two SFTENGs is shown in Figure 5a and b. In the SFTENGs, Metal 1 and 2 are placed in the same plane with a gap $g$ to form two electrodes. A freestanding layer with the same size of Metal 1 stands on the top of the metal electrodes and the distance between the bottom surface of freestanding layer and Metal 1 is defined as the freestanding height $h$. The width of this structure is defined as $w$. For dielectric SFTENGs shown in Figure 5a, the freestanding layer only contains a layer of dielectrics. And for metal SFTENGs, a thin layer of metal is deposited at the backside of the dielectric freestanding layer. These two structures look very similar, but unlike CFTENGs, the freestanding material will strongly influence the output characteristics of the SFTENGs.

The main working principle of SFTENGs is very similar to that of CFTENGs. The node concept can still be utilized to analyze their working principle. We first analyze dielectric SFTENGs. In contrast to CFTENGs, since the electrical potential of the dielectric bottom surface is not a constant, this surface could not be seen as a single node. The electric potential superposition principle is utilized to solve this problem. First, we assume that only a small region of $\mathrm{d} k$ in the bottom dielectric surface (the distance of this region to the left edge of the bottom dielectric surface is $k$ ) contains the tribo-charges with a density of $-\sigma$, and correspondingly the total charges on metal 1 and 2 are $\sigma w \mathrm{~d} k$. Utilizing the similar derivation method we utilized above, the total charges on metal 1 and $2\left(\mathrm{~d} Q_{1}\right.$ and $\left.\mathrm{d} Q_{2}\right)$ under $\mathrm{SC}$ conditions because of these tribo-charges can be given by the following equations, where $C_{\mathrm{i}}(k)$ states for the capacitance between this small surface $\sigma w \mathrm{~d} k$ and metal i: (For a detailed derivation, see ESI, Section 3) 


$$
\mathrm{d} Q_{1}=\frac{\sigma w \mathrm{~d} k}{1+\frac{C_{2}(k)}{C_{1}(k)}}(21 a) ; \quad \mathrm{d} Q_{2}=\frac{\sigma w \mathrm{~d} k}{1+\frac{C_{1}(k)}{C_{2}(k)}}
$$

Because of the superposition principle of the electrostatic field, the total charges on metal 1 and metal 2 considering the whole charges on the dielectric surface is the integration of each small tribo-charged region, which can be shown as:

$$
Q_{1}=\sigma w \int_{0}^{l} \frac{\mathrm{d} k}{1+\frac{C_{2}(k)}{C_{1}(k)}}(22 a) ; Q_{2}=\sigma w \int_{0}^{l} \frac{\mathrm{d} k}{1+\frac{C_{1}(k)}{C_{2}(k)}}(22 b)
$$

Therefore, $Q_{\mathrm{SC}, \text { final }}$ can be shown as:

$$
Q_{\mathrm{SC}, \text { final }}=\int_{0}^{l} \frac{\sigma w \mathrm{~d} k}{1+\left(\frac{C_{2}(k)}{C_{1}(k)}\right)_{x=g+l}}-\int_{0}^{l} \frac{\sigma w \mathrm{~d} k}{1+\left(\frac{C_{2}(k)}{C_{1}(k)}\right)_{x=0}}
$$

When $x=0$, the tribo-charged dielectric surface is much closer to metal 1 than to metal 2, so the ratio $C_{2}(k) / C_{1}(k)$ is close to 0 for all the $k$ values. Therefore, $Q_{1}$ will be approximately $\sigma w l$ and $Q_{2}$ will be approximately 0 from equation 22 . On the contrast, the ratio $C_{2}(k) / C_{1}(k)$ is close to infinity for all the $k$ values when $x=g+l$. Therefore, $Q_{1}$ will be approximately 0 and $Q_{2}$ will be approximately $\sigma w l$. Thus, $Q_{\mathrm{SC} \text {,final }}$ can reach $\sigma w l$ and the charge-transfer efficiency $\left(\eta_{\mathrm{CT}}\right)$ defined as the ratio between $Q_{\mathrm{SC} \text {,final }}$ and the total tribo-charges can reach $100 \%$. Same as CFTENGs, the change of the ratio of these two capacitances with the change of $x$ is the core-working principle of SFTENGs.

For metal SFTENGs, the basic electrostatic induction principle is the same as the dielectric SFTENGs. Therefore, as shown in Figure 5c, the numerically calculated trend of $Q_{S C}$ profile (under MACRS) is quite similar to that of dielectric SFTENGs. (Detailed numerical calculation parameter is listed in Table III). However, unlike CFTENGs, their characteristics of capacitance and $V_{\mathrm{OC}}$ are completely different from each other, as shown in the numerical results presented in Figure 5d and 5e. The capacitance profile of dielectric SFTENGs is nearly constant when the position of the freestanding layer changes. However, for metal SFTENGs, the capacitance when the freestanding layer is in the middle is several orders larger than that 
when the freestanding layer is at the two ends. This capacitance difference leads to the difference of their $V_{\mathrm{OC}}$ profile. Since the capacitance of metal-SFTENGs in the middle region is much larger than that of dielectric-SFTENGs, so from equation 8 , the open-circuit voltage of metal-SFTENGs in this region will be much smaller than that of dielectric-SFTENGs. And when $x$ is close to $g+l$, since the capacitance of the metal-SFTENG quickly dropped to the value close to the dielectric-SFTENG, the open-circuit voltage of the metal-SFTENG also quickly increase to a value close to the dielectric-SFTENG. Therefore, although the final open-circuit voltage values of the two SFTENGs are almost the same, the metal-SFTENG curve is much more non-linear than the dielectric-SFTENG curve and the maximum slope of the metal-SFTENG curve is much larger than that of the dielectric-SFTENG curve.

These differences in capacitance and $V_{\mathrm{OC}}$ profiles are mainly from the charge redistribution inside the metal freestanding layer. In dielectric-SFTENGs, the charges are not free to move. Since the relative dielectric constant of the commonly utilized dielectrics is not much larger than the relative dielectric constant of the air (always in the range of 2-4), the change of the capacitances due to the change of the freestanding-layer position is little. However, the charge on the surface of metal freestanding layer can redistribute to keep the electrical potential of the metal freestanding layer constant, which will lead to huge disturbance of the electrostatic field distribution. Therefore, the capacitance of metal-SFTENGs has two parts. The first part is the direct parasitic capacitance between these two electrodes, which can be analogous to the capacitance of dielectric SFTENGs. The second part is the serial connection of the capacitance between metal 1 and metal $3\left(C_{\mathrm{f} 1}\right)$ and metal 3 and metal $2\left(C_{\mathrm{f} 2}\right)$, which is the unique capacitance part of metal SFTENGs. When both the dielectric thickness and the freestanding height $h$ are not too large, the contribution of this serial connection is very significant to the total capacitance. At the two ends, either $C_{\mathrm{f} 1}$ or $C_{\mathrm{f} 2}$ will close to 0 because the metal freestanding layer only have overlapping region with one electrode, so the total capacitance of metal SFTENGs is close to the capacitance of dielectric SFTENGs. When $x$ 
equals to $(g+l) / 2, C_{\mathrm{f} 1}$ and $C_{\mathrm{f} 2}$ will be the same and their serial connection gets its maximum, leading to the largest total capacitance under this condition. At the same time, strong charge redistribution has been observed in the inner surface of the metal freestanding layer, as shown in Figure S5.

\subsection{Output characteristics}

Besides the basic output characteristics, the resistive load characteristics of SFTENGs have been studied as well. Same as CFTENGs, for SFTENGs with a resistive load, the equivalent circuit is still the same as Figure $3 \mathrm{a}[23]$ and the government equation combining their $V-Q-x$ relationship and Ohm's Law can be given by:[21]

$$
R \frac{d Q}{d t}=V=-\frac{1}{C} \times Q+V_{O C}
$$

The semi-analytical results of $V_{\mathrm{OC}^{-}} x$ and $C-x$ relationship inside equation 24 can be obtained from interpolation method of the FEM results.[21] Besides that, the motion process and the boundary condition must be specified to solve the above equation numerically. For the motion process, we still choose a harmonic vibration as a typical example, which is mathematically shown in Equation 25.

$$
x(\mathrm{t})=\frac{l+g}{2}\left(1-\cos \left(\frac{\pi v t}{l+g}\right)\right)
$$

Since the motion process is periodic, output from any initial boundary condition will gradually converge to a periodic output wave with the same period as the motion process after the first few periods, which is the steady-state.[24] The periodic boundary condition to obtain the steady-state is shown as:

$$
Q(t=0)=Q\left(t=\frac{2(l+g)}{v}\right)
$$

With the above periodic boundary condition, numerical calculation can be performed through the TENG simulator[23] and the results for the dielectric SFTENGs are plotted in Figure 6. Different from CFTENGs, the output is not a pure harmonic curve and contains some non- 
linear distortion because of the non-linear $V_{\mathrm{OC}^{-}}-x$ characteristics. However, this nonlinear distortion is not severe in the output curves because the capacitance is still quite close to a constant and the $V_{\mathrm{OC}^{-}} x$ curve is only non-linear at the two ends. When $R$ is small, the output is close to that of SC condition because of the small resistance of charge transport. As $R$ increases, its limitation to the charge transport between the two electrodes becomes more and more significant, resulting in a smaller oscillation magnitude of the charge transfer curve. When $R$ is very high, this oscillation magnitude reduces to close to 0 and the base of the charge transfer curve converge to half of the total tribo-charges. At the same time, the current also approaches to 0 while the voltage curves converge to a curve that is fully symmetric. It can be proved that this curve is the open-circuit voltage curve under symmetric charge reference state. (See ESI, Section 1 for discussion of symmetric charge reference state and its difference with MACRS) From this analysis, the SFTENGs also show the three-workingregion behavior, which is inherently because of the impedance match of $C$ and the load resistance. When $R$ is matched with the impedance of the TENG, the maximum energy from a single cycle can be reached. The optimum resistance from the calculation is $8.6 \mathrm{G} \Omega$, which is a bit lower than the impedance of the TENG capacitance under this frequency $(1 /(2 \pi f C)=$ 8.74 G $\Omega$ ). This small difference is because the non-linear $V_{\mathrm{OC}^{-}} x$ curve induces a few highfrequency components of the $V_{\mathrm{OC}}$ curve.

For the metal SFTENGs, similar numerical calculation was carried out and the results are shown in Figure S6. The change of the output curves with the load resistance is very similar to the dielectric SFTENGs and the three-working-region behavior is also observed in metal SFTENGs. However, in the metal SFTENGs, the capacitance is no longer a constant and the formed RC system is time-variant. Therefore, the distortion of the output curves from purely harmonic behavior is much more severe than those in the dielectric SFTENGs. Besides, at this time although the optimum resistance can still be thought as the "impedance" match between $C$ and the load resistance, there is no rigorous definition of the TENG's internal impedance 
because the system is now time-variant, leading to the result that no simple equation can be provided to estimate the optimum resistance. The optimum resistance value of metal SFTENGs is slightly smaller than that of dielectric SFTENGs because of their larger average capacitance. Finally, the optimum harvested energy of metal SFTENGs is a little higher than dielectric SFTENGs, which is because the higher non-linearity of the $V_{\mathrm{OC}}$ curve in the metalSFTENGs will generate a higher voltage peak value under the optimum resistance, as shown in Figure S7.

\subsection{Tolerance to the freestanding height}

The most significant advantage of the SFTENG structure is its excellent tolerance to the freestanding height $h$. This loosens the requirement of the sliding object and reduces the energy loss due to the friction. The influence of the freestanding height on the output performance of dielectric SFTENGs is first investigated. Besides the parameter specified in the figure, the value of all the other parameters are the same as listed in Table III. From Figure 7a, $Q_{\mathrm{SC} \text {,final }}$ decreases when $h$ increases, which is mainly because of the reduce of the difference between $\left(C_{2}(k) / C_{1}(k)\right)_{x=0}$ and $\left(C_{2}(k) / C_{1}(k)\right)_{x=g+l}$. Specifically, when $h=0, C_{2}(k) / C_{1}(k)$ equals to 0 at the position of $x=0$ and equals to infinity at the position of $x=g+l$, so $Q_{\mathrm{SC} \text {,final }}$ can reach $\sigma w l$. When $h$ is large enough, the average distance between the tribo-charge surface and two electrodes will be approximately the same. Thus both $\left(C_{2}(k) / C_{1}(k)\right)_{x=0}$ and $\left(C_{2}(k) / C_{1}(k)\right)_{x=g+l}$ are approximately 1 , resulting in a zero $Q_{\text {sc,final }}$. But the decreasing slope with height is much flatter than the attached-electrode STENG case. For a traditional attached-electrode sliding-mode TENG as shown in Figure S8,[21] since electrode 1 is attached with the tribo-charged surface, $C_{1}(k)$ is always very large. Therefore, when there is a height between the tribo-charged surface and electrode 2 which is larger than the dielectric thickness, $C_{2}(k)$ will be always smaller than $C_{1}(k)$. Therefore, both $\left(C_{2}(k) / C_{1}(k)\right)_{x=0}$ and $\left(C_{2}(k) / C_{1}(k)\right)_{x=g+l}$ is close to 0 and $Q_{\mathrm{SC}, \text { final }}$ is strongly reduced. From the numerical calculation 
results, the half-life height that $Q_{\mathrm{SC} \text {,final }}$ reduces to its half-value is $250 \mu \mathrm{m}$, while that for SFTENG structure is about $2 \mathrm{~cm}$. This excellent height tolerance of SFTENGs has also been experimentally proven in our previous report.[16] As for the capacitance profile shown in Figure $7 \mathrm{~b}$, the increase of $h$ only slightly decreases the total capacitance since the capacitance in the dielectric SFTENGs is mainly from the direct capacitance between metal 1 and metal 2. For the $V_{\mathrm{OC}}$ profile of the dielectric SFTENG shown in Figure 7c, it almost has the same trend with $Q_{\mathrm{SC}}$, for the capacitance almost has no dependence with $h$. As for the total energy- $R$ curve, this degradation of both $Q_{\mathrm{SC}}$ and $V_{\mathrm{OC}}$ will lead to the decrease of the maximum harvested energy when $h$ increases. However, the optimum resistance stays almost the same, which is because of the nearly same inherent capacitance for different $h$.

For metal SFTENGs, their height characteristics have some similarity with dielectric SFTENGs, but it will still have several differences. Since the basic electrostatic induction mechanism in the metal SFTENG system is very similar to that of dielectric SFTENG system, the change of $Q_{\mathrm{SC}}$ profiles with height is similar to that of dielectric SFTENG system. However, there are still many differences. When $h$ increases, the influence of the metal layer on the electrostatic system reduces and both $C_{\mathrm{f} 1}$ and $C_{\mathrm{f} 2}$ significantly decrease. Thus, as shown in Figure 8b, the total capacitance at the middle position is largely affected when $h$ first increases from 0 . When $h$ is large enough, the total capacitance starts to be dominated by the direct parasitic capacitance between the two electrodes and is nearly independent of $x$. At this time, the metal SFTENGs are indistinguishable with the dielectric SFTENGs. As for the open-circuit voltage shown in Figure 8c, the metal SFTENGs also show a very complicated profile, which is because both $Q_{\mathrm{SC}}$ and $C$ have strong dependence on the freestanding height. $V_{\mathrm{OC}-\text { final }}$ will decrease because of a reduced $Q_{\mathrm{SC}-\text { final }}$ and an almost non-changed $C$ in that region. However, at the middle region, $V_{\mathrm{OC}}$ will increase first when $h$ increases then decreases when $h$ is already large enough. This is because the decreasing speed of the serial connection 
of $C_{\mathrm{f} 1}$ and $C_{\mathrm{f} 2}$ is faster than the decreasing speed of $Q_{\mathrm{SC}}$ in this region. When $h$ is small and $x$ is in the middle range, this serial connection dominates the total capacitance and $V_{\mathrm{OC}}$ has this unusual increase. As for the total harvested energy, it also has the similar decay trend as the dielectric SFTENGs. However, since the average total capacitance drops with the increase of $h$, the optimum resistance shifts to the right when $h$ first increases and finally stays on the value which is almost the same as the dielectric SFTENGs. Besides, since the metal SFTENGs will be more close to the dielectric SFTENGs when $h$ increases, the increase of total harvested energy due to the non-linear metal SFTENGs as discussed above will also decrease. The tolerance of height for metal SFTENGs is a little worse than dielectric SFTENGs.

\subsection{Influence of gap between electrodes}

Besides the freestanding height $h$, another important design parameter is the gap distance between the electrodes $(g)$. The capacitance between the electrodes is strongly dependent on the gap distance. Therefore, the gap distance will have strong effect on the total output characteristics.

The effect of $g$ on the total output performance for dielectric SFTENGs under $h=0$ conditions is studied first and plotted in Figure 9. Besides the parameters specified in the figure, all the other parameters is the same as listed in Table III. Since $h$ equals to 0 , the ratio $C_{2}(k) / C_{1}(k)$ is still 0 when $x=0$ and infinity when $x=g+l$. Therefore, the charge transfer efficiency $\eta_{\mathrm{CT}}$ can still reach $100 \%$ for all the $g$ values and $Q_{\text {SC-final }}$ will not change with $g$. However, the capacitance between the two electrodes decreases when $g$ increases, as shown in Figure $9 \mathrm{~b}$. Thus, through equation $8, V_{\mathrm{OC}-\text { final }}$ when a full separation is reached will increase with $g$. Therefore, considering the total energy harvested in one cycle, the dielectric SFTENG with a higher $g$ will generate a larger total energy with a higher optimum resistance. However, for dielectric SFTENGs with a higher $g$, their cycle $(T)$ is also longer when the average 
velocity stays the same. Therefore, the average power (defined as equation 27 a) will have a different trend with the total harvested energy, as shown in Figure 9d.

$$
P_{\text {avg }}=\frac{R \int_{0}^{T} I^{2} d t}{T}(27 a) \quad T=\frac{2(l+g)}{v}
$$

When $g$ first increases from 0 , the cycle length increases only a little because $l$ still dominates the total cycle length. Therefore, the increase of $V_{\mathrm{OC}-\text { final }}$ dominates the total average power and average power increases with $g$. However, when $g$ is already very large, the decreasing slope of the total capacitance is flattened. Therefore, the increase of $V_{\text {OC-final }}$ cannot catch up with the increasing speed of $T$ and the average power begins to drop. Thus, an optimum gap is observed to generate the largest average power.

For metal SFTENGs under $h=0$ conditions, similar results have also been observed, as shown in Figure 10. The charge transfer efficiency $\eta_{\mathrm{CT}}$ can still reach $100 \%$ for all the $g$ values and $Q_{\text {SC-final }}$ will not change with $g$. The total capacitance of metal SFTENGs also decrease with the increase of $g$, but this decrease contributes from two reasons. The first reason is the same as dielectric SFTENGs, which will lead to the decrease of the direct parasitic capacitance between these two electrodes. Besides, when $g$ increases, the region when the freestanding layer are overlapped with both of the two electrodes decreases, leading a decrease of the serial connection of $C_{\mathrm{f} 1}$ and $C_{\mathrm{f} 2}$. When $g$ is larger than $l$, the region that the freestanding layer is overlapped with both electrodes no longer exists and the serial connection of $C_{\mathrm{f} 1}$ and $C_{\mathrm{f} 2}$ can be neglected. The influence of $g$ on $V_{\mathrm{OC}}$ of metal SFTENGs is very similar to the case in dielectric SFTENGs. $V_{\text {OC-final }}$ will increase with the increase of $g$. Also, similar to the dielectric SFTENGs, the optimum resistance still increases when $g$ increases and there is an optimum gap to reach the highest average power.

Besides the $h=0$ case we discussed above, the influence of the gap distance will be much 
more complicated if there is a non-zero freestanding height. When $h$ is non-zero, the capacitance ratio $C_{2}(k) / C_{1}(k)$ is no longer 0 when $x=0$ and no longer infinity when $x=g+l$. And if the gap decreases, the difference of the capacitance ratio $C_{2}(k) / C_{1}(k)$ between $x=0$ position and $x=g+l$ position will reduce as a response, resulting in a decrease of $Q_{\text {SC-final }}$. The 3d plot of $Q_{\text {SC-final }}$ under different $h$ and $g$ for dielectric SFTENG is shown in Figure 11a and its 2D injection is shown in Figure S9. Consistent with our theoretical analysis, when $g$ increases, the slope of $Q_{\mathrm{SC}-\mathrm{final}}-h$ curve decreases and the dielectric SFTENG has more tolerance to the height $h$. In addition, $Q_{\text {sC-final }}$ will increase with the increase of $g$ when $h$ is a non-zero constant and the slope of $Q_{\mathrm{SC}_{\text {-final }}} g$ curves increase when $h$ goes up. As for the average power shown in Figure 11b, there is still an optimum gap value when $h$ increases. This optimum gap at which the highest average power is reached increases with $h$, as a result of better tolerance of height for larger gap devices. For metal SFTENGs, similar results have also been observed, as shown in Figure 11c and d. However, the optimum gap increase in the metal SFTENGs with the freestanding height is faster than in the dielectric SFTENGs.

\section{Conclusion}

In summary, the theoretical basis of freestanding triboelectric nanogenerators is presented in detail. Based on the charge separation mechanism, freestanding triboelectric nanogenerators have two categories: contact-mode freestanding triboelectric nanogenerators (CFTENGs) based on vertical charge separation and sliding-mode freestanding triboelectric nanogenerators (SFTENGs) based on in-plane charge separation. For both of them, the change of the capacitance ratio between the tribo-charged surface and two electrodes with the position of the freestanding layer is the core working mechanism of freestanding triboelectric nanogenerators. CFTENGs have inherent superior linear characteristics. These superior linear characteristics are highly desired to harvest vibration energy in non-contact working mode and realize accurate detection of vibration without non-linear distortion. SFTENGs have two 
sub-categories based on the material of their freestanding layer. The inherent capacitance of dielectric SFTENGs is nearly constant while that of metal SFTENGs varies with the position of the freestanding layer. Their most significant advantage is their superior tolerance of the freestanding height, which can ensure their high performance working in the non-contact mode. Increasing the electrode gap will increase the open-circuit voltage and enhance their tolerance to the height. Besides, an optimum electrode gap is observed to generate the maximum average power and this optimum electrode gap increases with the freestanding height. The theoretical work here is the first in-depth analysis of the working principle of freestanding TENGs. The discussion about the output characteristics, structural design and optimization can serve as a guideline for rational design to maximize electrical output for energy harvesters and sensitivity for self-powered sensors.

\section{Acknowledgements}

S. Niu, Y. Liu, X. Chen contribute equally to this work. Research was supported by U.S. Department of Energy, Office of Basic Energy Sciences (Award DE-FG02-07ER46394), MANA, National Institute For Materials Science, Japan, a joint project with Sungkyunkwan University, Korea, the Hightower Chair foundation, and the "thousands talents" program for pioneer researcher and his innovation team, China.

[1] S.P. Beeby, R.N. Torah, M.J. Tudor, P. Glynne-Jones, T. O'Donnell, C.R. Saha, S. Roy, J. Micromech. Microeng., 17 (2007) 1257-1265.

[2] C.R. Saha, T. O'Donnell, N. Wang, R. McCloskey, Sens. Actuators A, 147 (2008) 248253.

[3] Y. Suzuki, IEEJ Trans. Electr. Electron., 6 (2011) 101-111.

[4] H.W. Lo, Y.C. Tai, J. Micromech. Microeng., 18 (2008) 104006.

[5] P. Basset, D. Galayko, A.M. Paracha, F. Marty, A. Dudka, T. Bourouina, J. Micromech. Microeng., 19 (2009) 115025.

[6] Y.B. Xie, D. Bos, L.J. de Vreede, H.L. de Boer, M.J. van der Meulen, M. Versluis, A.J. Sprenkels, A. van den Berg, J.C.T. Eijkel, Nat Commun, 5 (2014) 3575.

[7] H.B. Fang, J.Q. Liu, Z.Y. Xu, L. Dong, L. Wang, D. Chen, B.C. Cai, Y. Liu, Microelectron J, 37 (2006) 1280-1284. 
[8] R.G. Horn, D.T. Smith, A. Grabbe, Nature, 366 (1993) 442-443.

[9] H.T. Baytekin, A.Z. Patashinski, M. Branicki, B. Baytekin, S. Soh, B.A. Grzybowski, Science, 333 (2011) 308-312.

[10] Y.S. Zhou, S.H. Wang, Y. Yang, G. Zhu, S.M. Niu, Z.H. Lin, Y. Liu, Z.L. Wang, Nano Lett., 14 (2014) 1567-1572.

[11] L.S. McCarty, G.M. Whitesides, Angew. Chem. Int. Ed., 47 (2008) 2188-2207.

[12] F.R. Fan, Z.Q. Tian, Z.L. Wang, Nano Energy, 1 (2012) 328-334.

[13] S.H. Wang, L. Lin, Y.N. Xie, Q.S. Jing, S.M. Niu, Z.L. Wang, Nano Lett., 13 (2013) 2226-2233.

[14] S. Kim, M.K. Gupta, K.Y. Lee, A. Sohn, T.Y. Kim, K.S. Shin, D. Kim, S.K. Kim, K.H. Lee, H.J. Shin, D.W. Kim, S.W. Kim, Adv. Mater., 26 (2014) 3918-3925.

[15] X.S. Zhang, M.D. Han, R.X. Wang, B. Meng, F.Y. Zhu, X.M. Sun, W. Hu, W. Wang, Z.H. Li, H.X. Zhang, Nano Energy, 4 (2014) 123-131.

[16] S.H. Wang, Y.N. Xie, S.M. Niu, L. Lin, Z.L. Wang, Adv. Mater., 26 (2014) 2818-2824.

[17] G. Zhu, J. Chen, T.J. Zhang, Q.S. Jing, Z.L. Wang, Nat Commun, 5 (2014) 3426.

[18] L. Lin, S.H. Wang, S.M. Niu, C. Liu, Y.N. Xie, Z.L. Wang, Acs Appl Mater Inter, 6 (2014) 3038-3045.

[19] Y.N. Xie, S.H. Wang, S.M. Niu, L. Lin, Q.S. Jing, J. Yang, Z.Y. Wu, Z.L. Wang, Adv. Mater., 26 (2014) 6599-6607.

[20] S.M. Niu, Y. Liu, S.H. Wang, L. Lin, Y.S. Zhou, Y.F. Hu, Z.L. Wang, Adv. Funct. Mater., 24 (2014) 3332-3340.

[21] S.M. Niu, Y. Liu, S.H. Wang, L. Lin, Y.S. Zhou, Y.F. Hu, Z.L. Wang, Adv. Mater., 25 (2013) 6184-6193.

[22] S.M. Niu, S.H. Wang, L. Lin, Y. Liu, Y.S. Zhou, Y.F. Hu, Z.L. Wang, Energy Environ. Sci., 6 (2013) 3576-3583.

[23] S.M. Niu, Y.S. Zhou, S.H. Wang, Y. Liu, L. Lin, Y. Bando, Z.L. Wang, Nano Energy, 8 (2014) 150-156.

[24] S.M. Niu, S.H. Wang, Y. Liu, Y.S. Zhou, L. Lin, Y.F. Hu, K.C. Pradel, Z.L. Wang, Energy Environ. Sci., 7 (2014) 2339-2349.

(a)

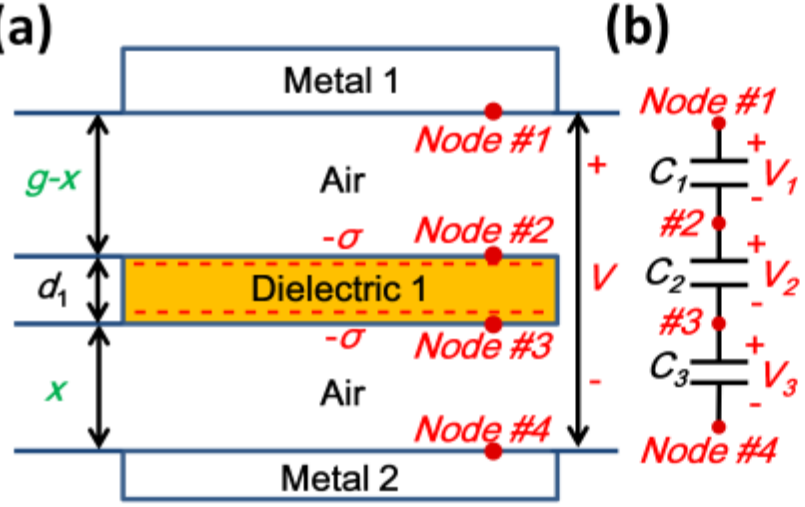

(c)

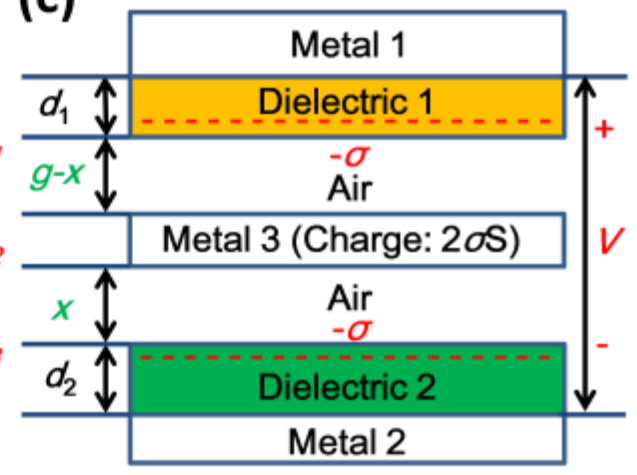

Figure 1. Theoretical models of CFTENGs. (a) model of a typical dielectric freestanding layer CFTENG. (b) equivalent circuit model of the dielectric CFTENG electrostatic system. (c) model of a typical metal freestanding layer CFTENG. 
(a)

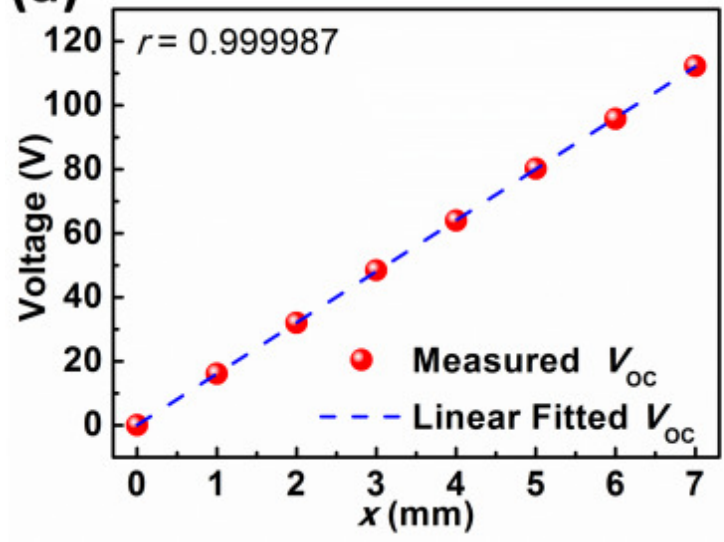

(b)

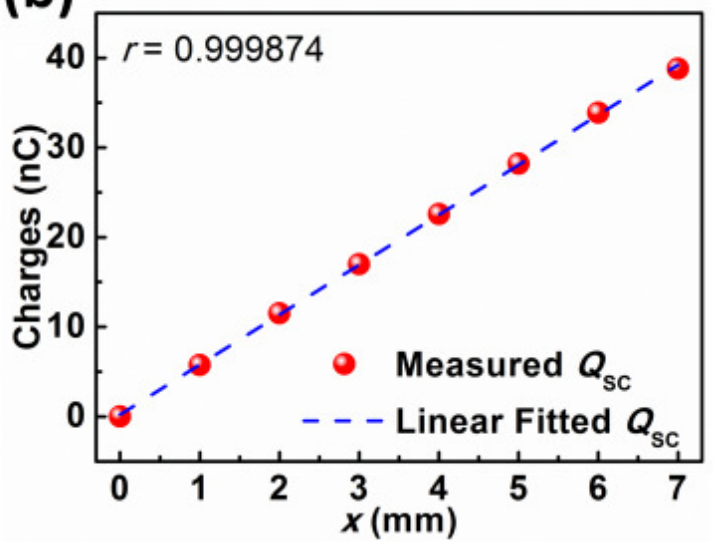

Figure 2. Experimental measured (a) $V_{\mathrm{OC}}$ and (b) $Q_{\mathrm{SC}}$ of CFTENGs. Both of them show accurate linear relationship with $x$.

(a)

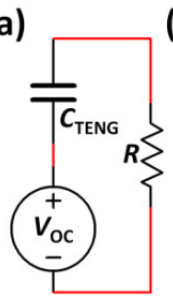

(b)

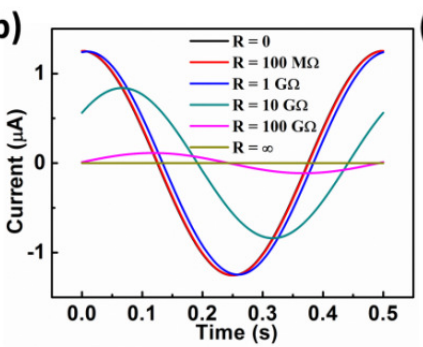

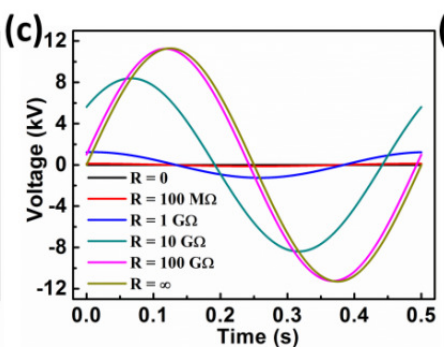

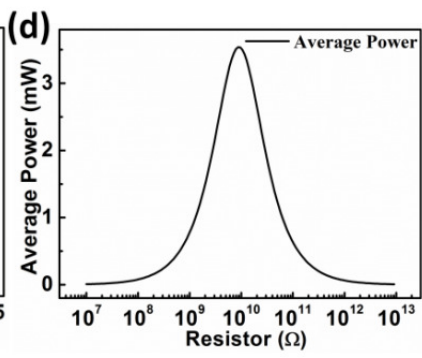

Figure 3. Basic output characteristics of CFTENGs. (a) Equivalent circuit model when a CFTENG is connected with a resistive load. (b) Current-time relationship at different load resistances. (c) Voltage-time relationship at different load resistances. (d) The dependence of the average power output on the load resistance. 

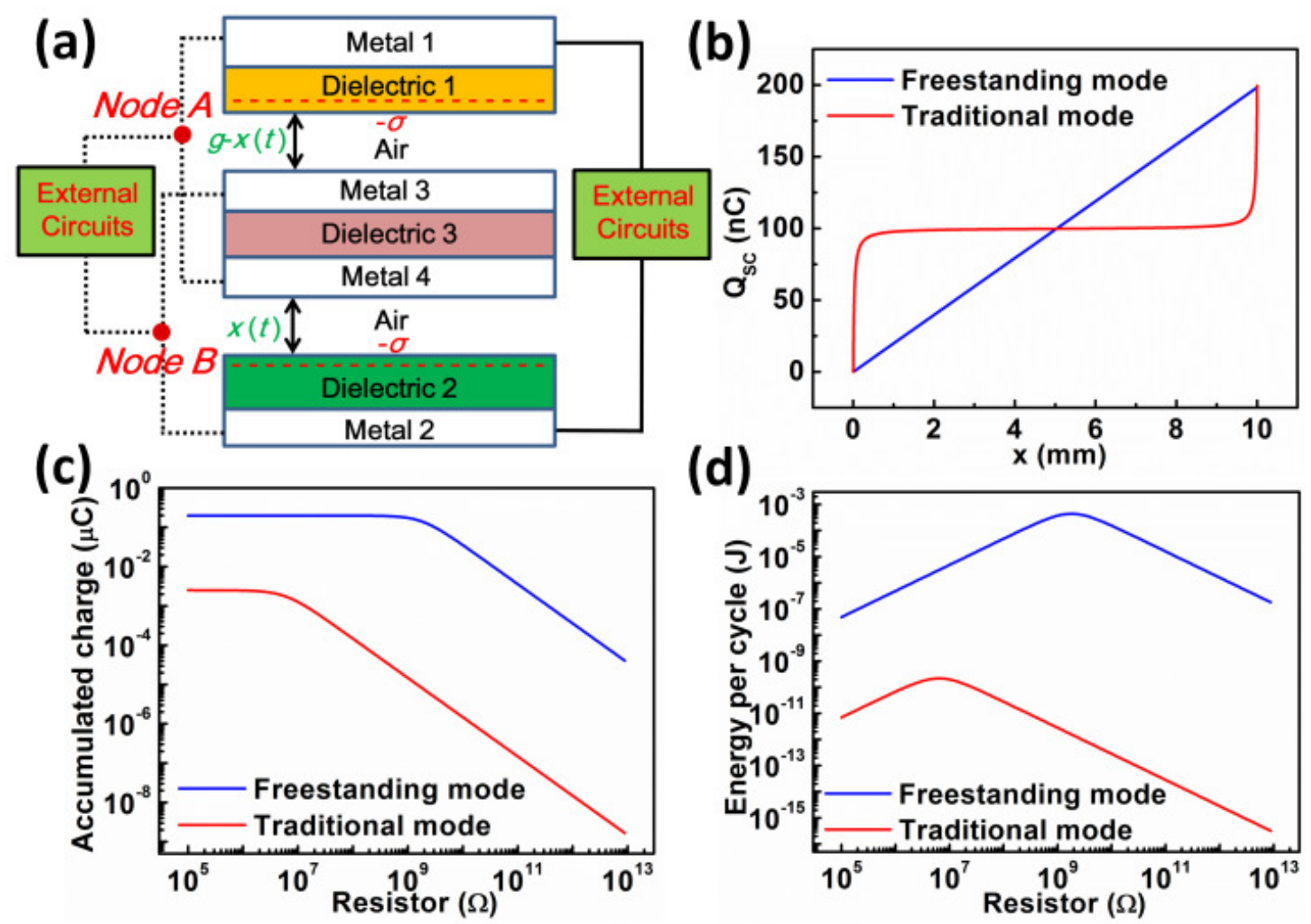

(d)

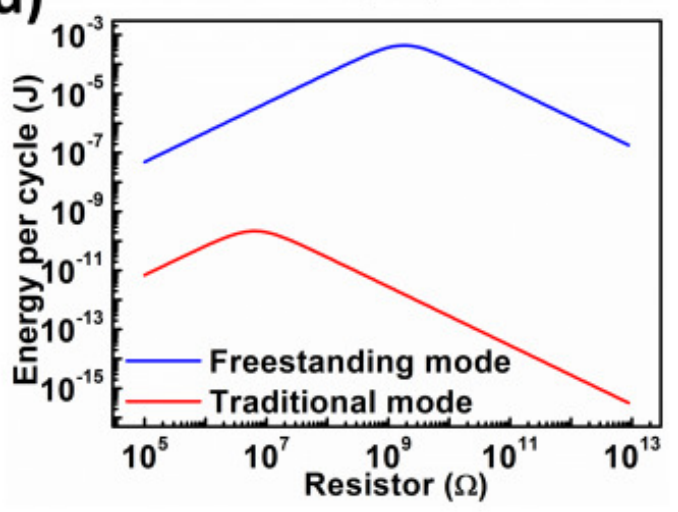

Figure 4. The advantages of CFTENGs in harvesting vibration energy compared to tradition two attached-electrode TENGs. (a) Structure of the carefully designed TENG which can work in both freestanding mode and traditional attached-electrode mode. (b) Compare of shortcircuit transferred charges characteristics under these two modes. (c-d) When the vibration amplitude is only a quarter of the air gap, the comparison of these two modes considering the amount of the transferred charges and harvested vibration energy in one cycle under different load resistance. 

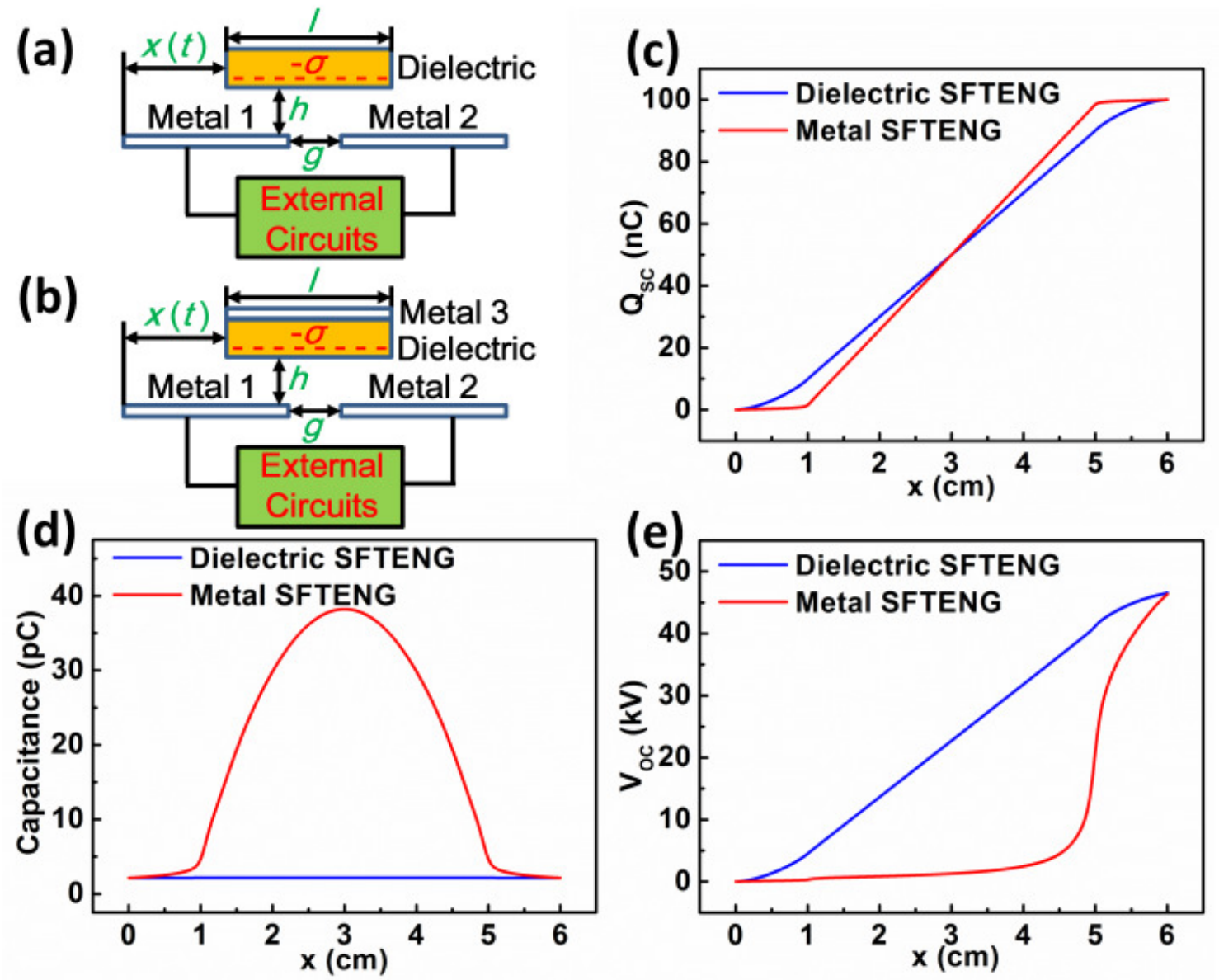

Figure 5. Models and basic output characteristics of SFTENGs. (a) FEM model of a typical dielectric SFTENG. (b) FEM model of a typical metal SFTENG. (c-e) Influence of freestanding layer material on the basic output characteristics: (c) short-circuit transferred charges under MACRS, (d) total capacitance between the two electrodes, and (e) open-circuit voltage under MACRS. 

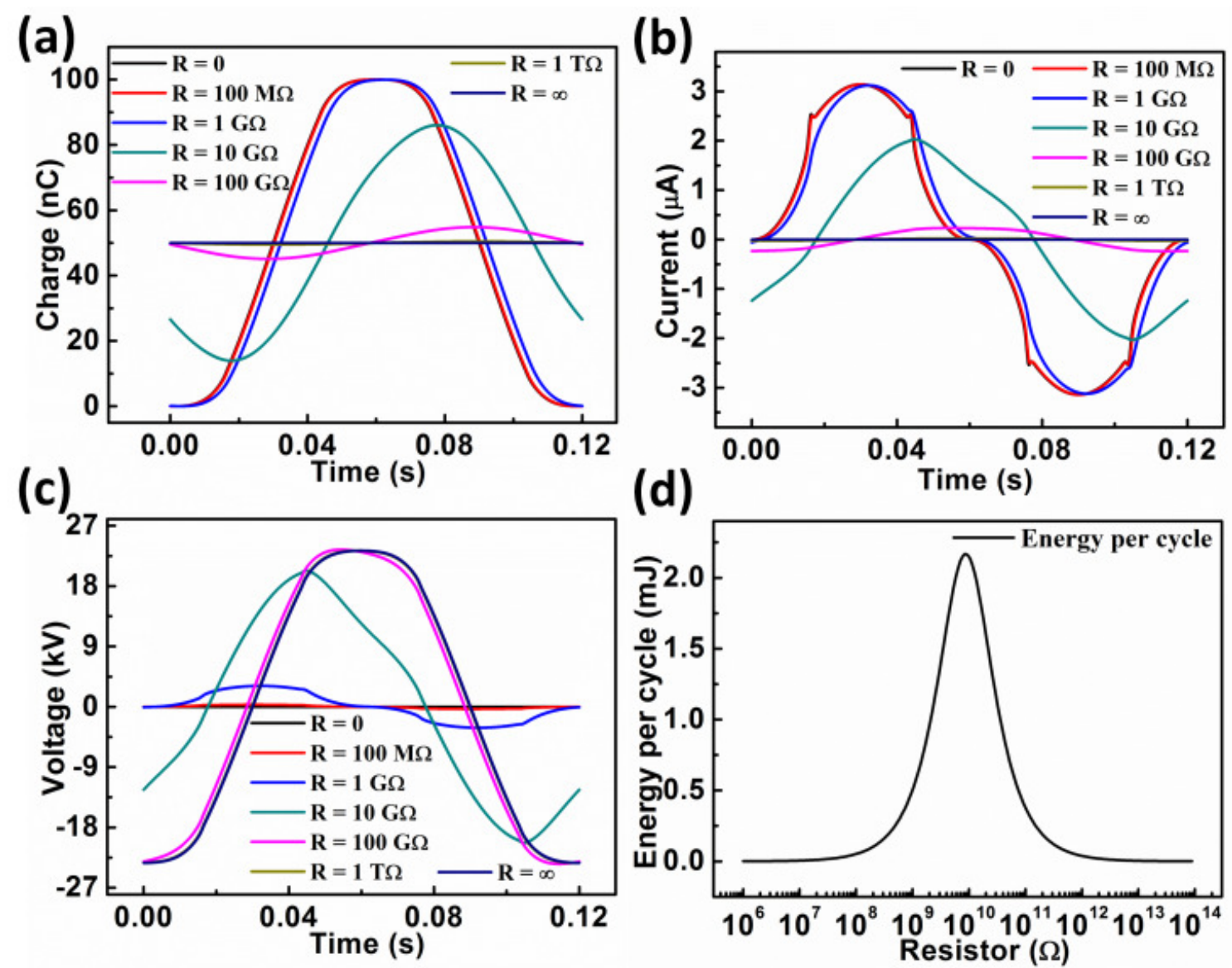

Figure 6. Output properties of dielectric SFTENGs under single frequency harmonic vibration. (a) Transferred charge-time relationship at different load resistances. (b) Currenttime relationship at different load resistances. (c) Voltage-time relationship at different load resistances. (d) The dependence of the total energy harvested in a single period on the load resistance. 

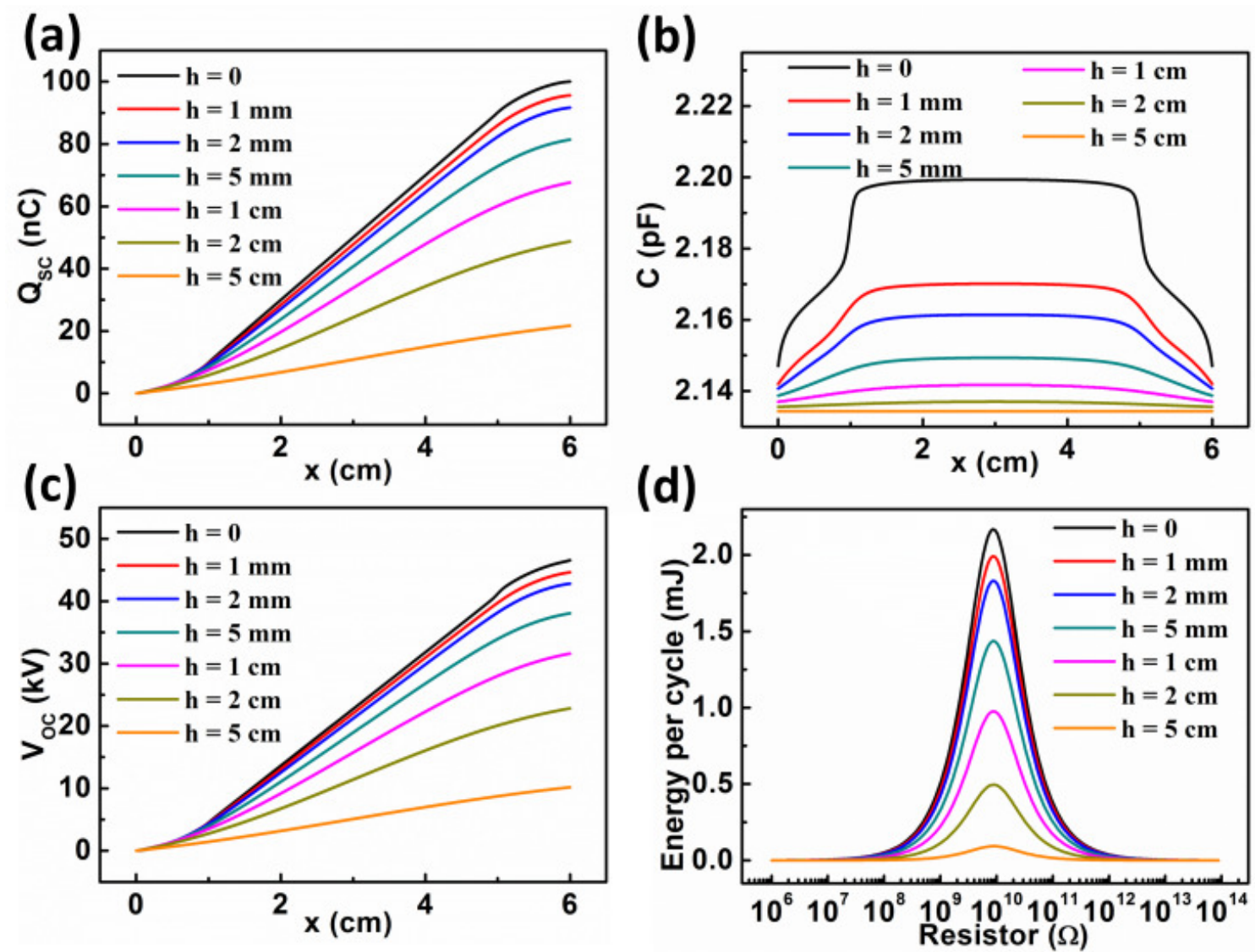

Figure 7. Tolerance of height for dielectric SFTENGs. (a-c) Influence of the freestanding height $h$ on (a) $Q_{\mathrm{SC}}$ curves under MACRS, (b) total capacitance curves, and (c) $V_{\mathrm{OC}}$ curves under MACRS of the dielectric SFTENG. (d) The harvested energy by the dielectric SFTENG in one cycle under different load resistance and different $h$. 

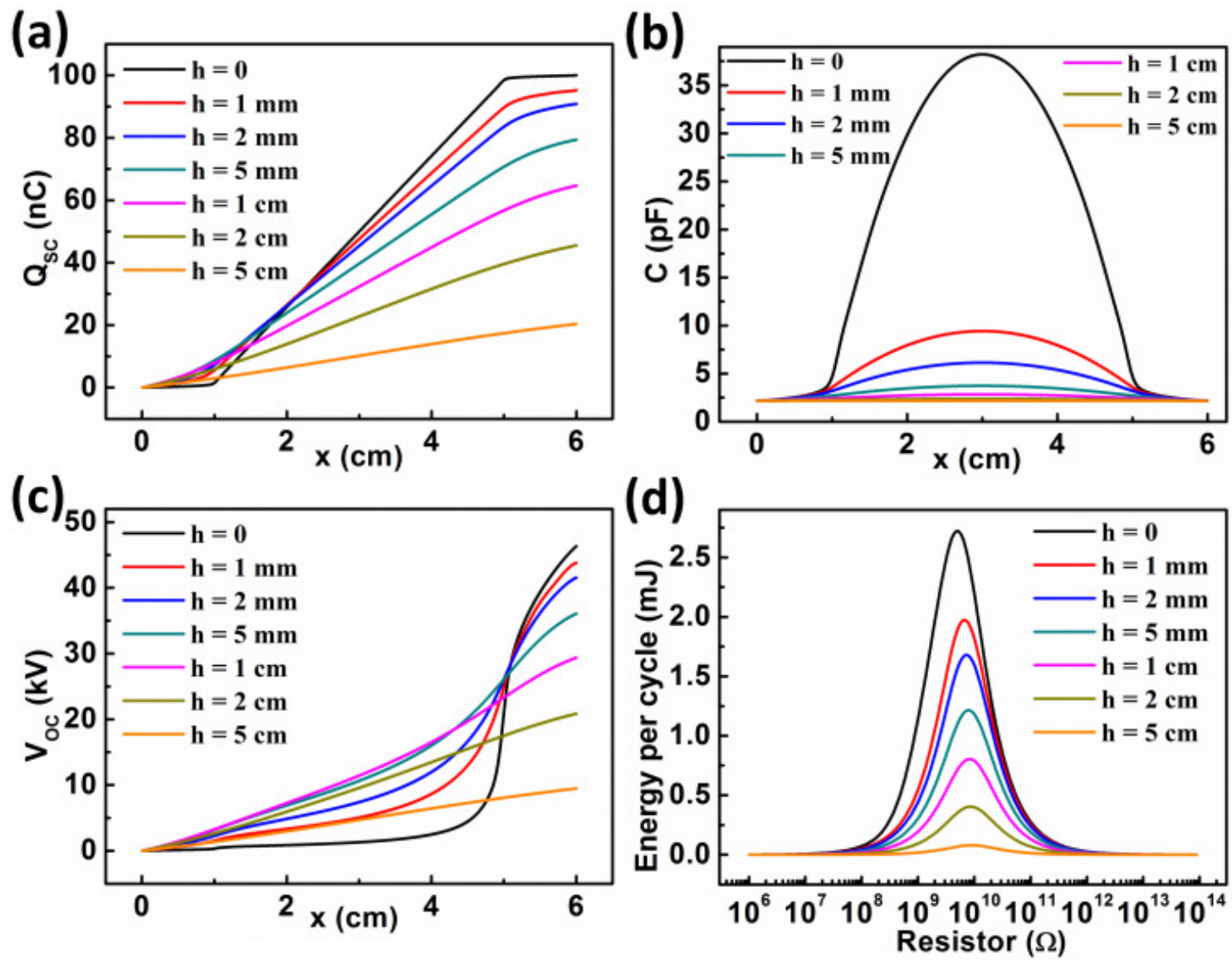

Figure 8. Tolerance of height for metal SFTENGs. (a-c) Influence of the freestanding height $h$ under $g=1 \mathrm{~cm}$ condition on (a) $Q_{\mathrm{SC}}$ curves under MACRS, (b) total capacitance curves, and (c) $V_{\text {OC }}$ curves of the metal SFTENG under MACRS. (d) The harvested energy by the metal SFTENG in one cycle under different load resistance and different $h$. 

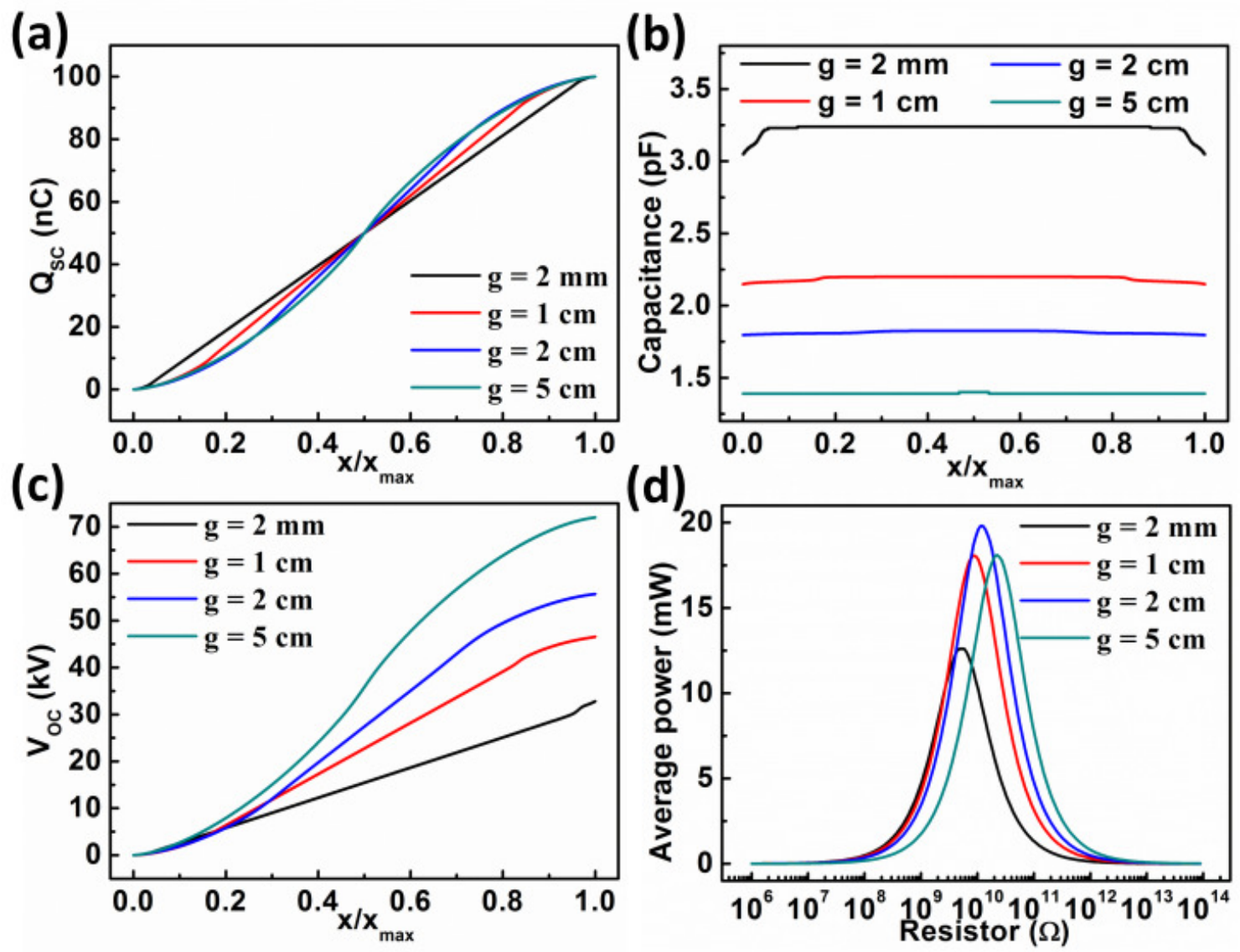

Figure 9. Influence of the gap in dielectric SFTENGs under $h=0$ condition. (a-c) Influence of the electrode gap $g$ under $h=0$ condition on (a) $Q_{\text {SC }}$ curves under MACRS, (b) total capacitance curves, and (c) $V_{\mathrm{OC}}$ curves under MACRS of the dielectric SFTENG. (d) The average power by the dielectric SFTENG in one cycle under different load resistance and different $g$. 

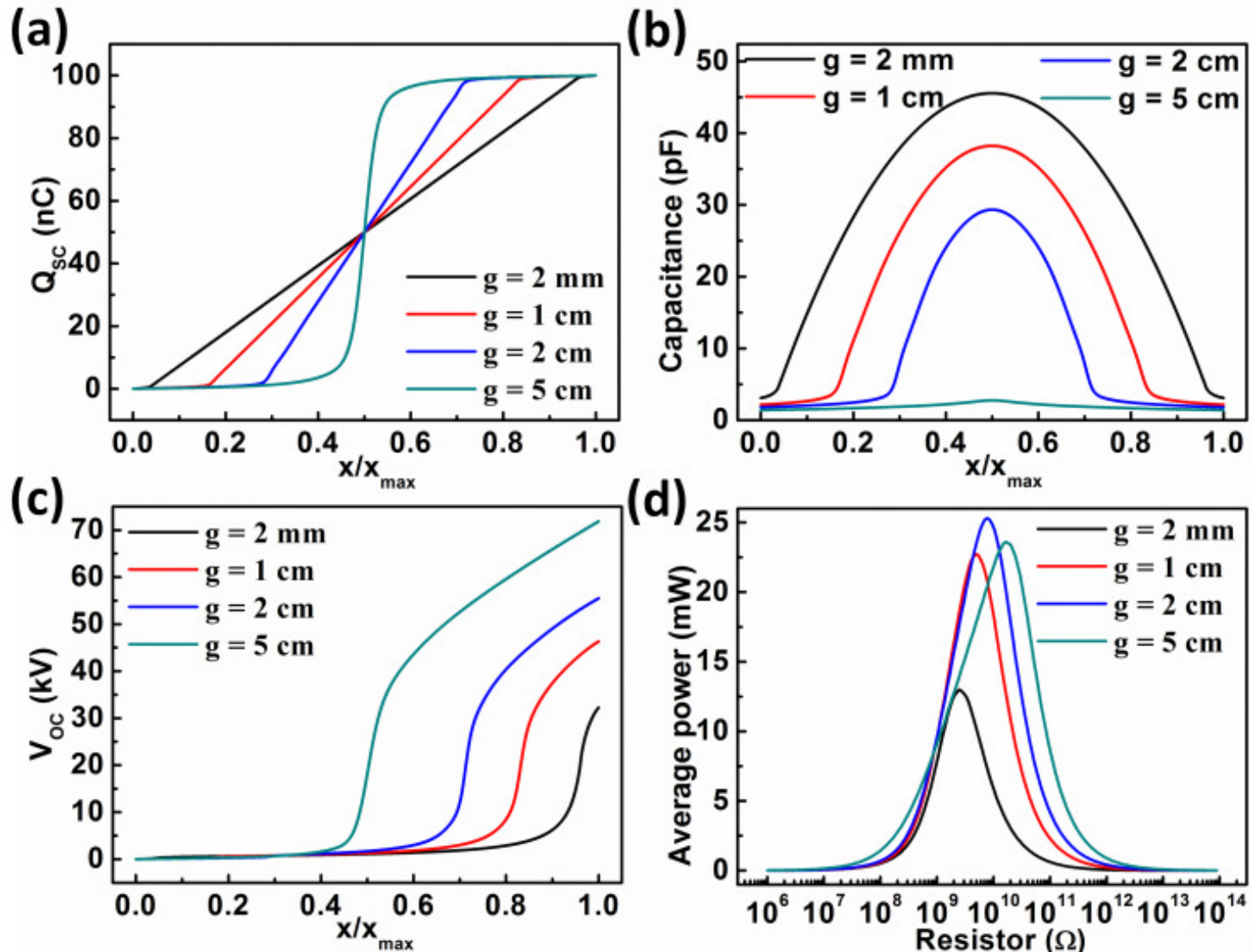

Figure 10. Influence of the gap in metal SFTENGs under $h=0$ condition. (a-c) Influence of the electrode gap $g$ under $h=0$ condition on (a) $Q_{\text {SC }}$ curves under MACRS, (b) total capacitance curves, and (c) $V_{\mathrm{OC}}$ curves under MACRS of the metal SFTENG. (d) The average power by the metal SFTENG in one cycle under different load resistance and different $g$. 

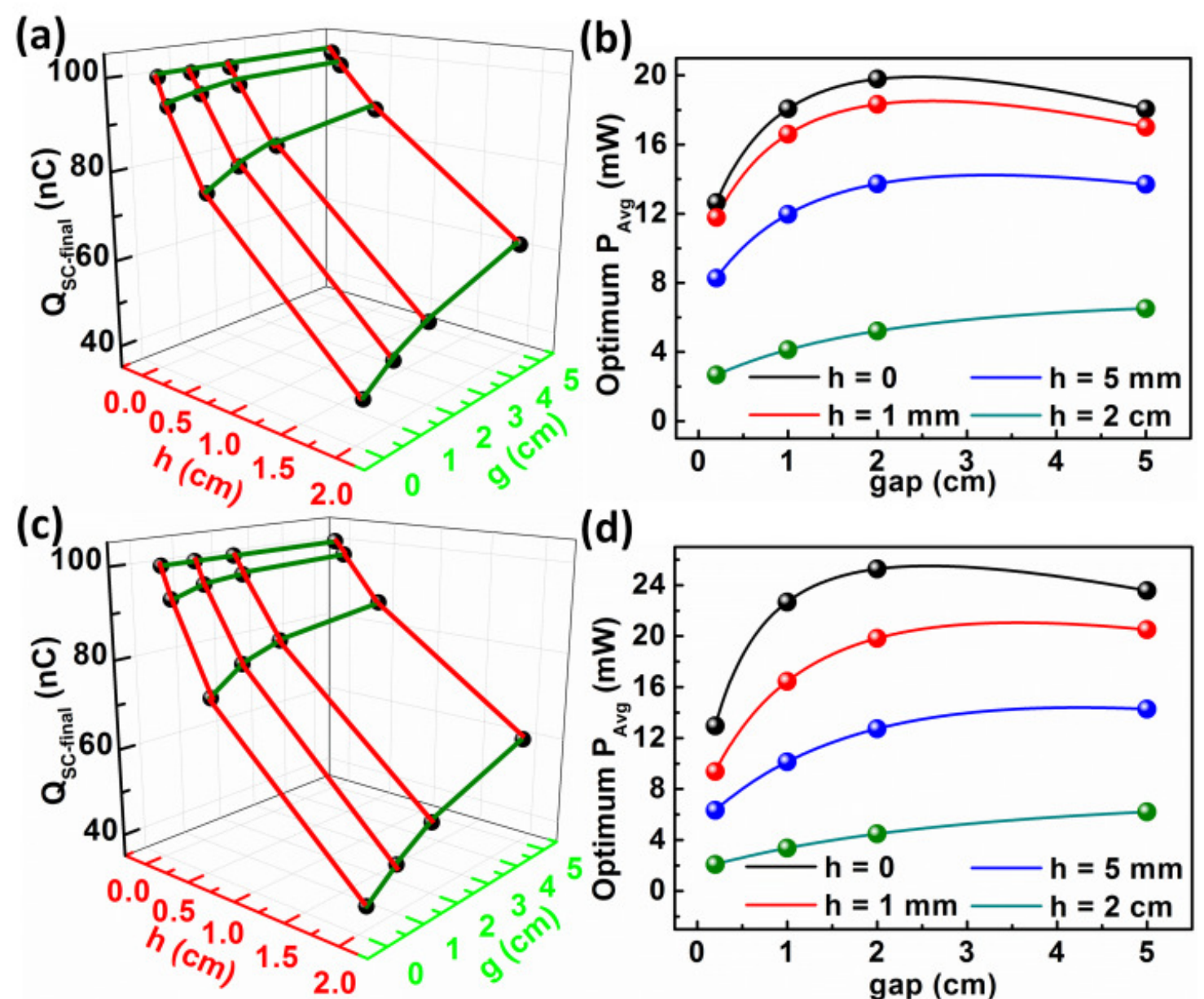

(d)

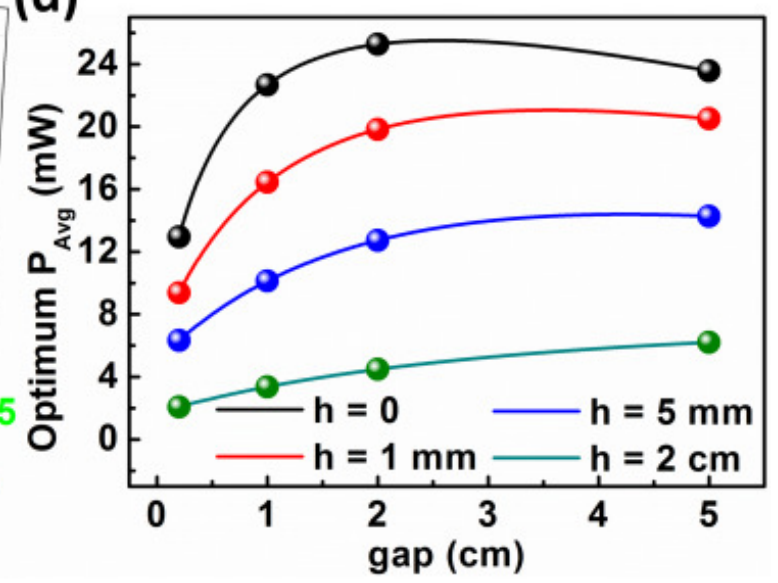

Figure 11. Coupling effect of gap and height on dielectric SFTENGs and metal SFTENGs. (a) Dependence of the short circuit transferred charge of the dielectric SFTENG when a full separation is reached on the freestanding height and the electrode gap. (b) Influence of the freestanding height on the optimum gap of the dielectric SFTENG at which the maximum average power is reached. (c) Dependence of the short circuit transferred charge of the metal SFTENG when a full separation is reached on the freestanding height and the electrode gap. (d) Influence of the freestanding height on the optimum gap of the metal SFTENG at which the maximum average power is reached.

Table 1. Parameter utilized in the output characteristic calculation of CFTENGs

\begin{tabular}{cc}
\hline \hline Structure Component & Parameter Utilized \\
\hline Dielectric 1 & $d_{l}=50 \mu \mathrm{m}, \varepsilon_{r l}=2.1$ \\
Area size of the dielectrics $S$ & $100 \mathrm{~cm}^{2}$ \\
Air gap distance $g$ & $1 \mathrm{~cm}^{-2}$ \\
Tribo-charge surface density $\sigma$ & $10 \mu \mathrm{Cm}^{-2}$ \\
Vibration angular frequency $\omega$ & $4 \pi \mathrm{s}^{-1}$ \\
Vibration amplitude $A_{0}$ & $5 \mathrm{~mm}$ \\
Vibration central position $x_{0}$ & $5 \mathrm{~mm}$ \\
\hline \hline
\end{tabular}

Table 2. Parameter utilized in the comparison of output characteristics between freestanding mode and traditional attached-electrode mode 


\begin{tabular}{cc}
\hline \hline Structure Component & Parameter Utilized \\
\hline Dielectric 1, Dielectric 2 & $d_{l}=d_{2}=50 \mu \mathrm{m}, \varepsilon_{r l}=\varepsilon_{r 2}$ \\
Dielectric 3 & $=2.1$ \\
Area size of the dielectrics $S$ & $d_{3}=125 \mu \mathrm{m}, \varepsilon_{r 3}=3.4$ \\
Air gap distance $g$ & $100 \mathrm{~cm}^{2}$ \\
Tribo-charge surface density $\sigma$ & $1 \mathrm{~cm}^{-2}$ \\
Vibration angular frequency $\omega$ & $10 \mu \mathrm{Cm}^{-2}$ \\
\hline \hline
\end{tabular}

Table 3. Parameter utilized in the calculation of output characteristics for both dielectric and metal SFTENGs

\begin{tabular}{cc}
\hline \hline Structure Component & Parameter Utilized \\
\hline Dielectric & $d=500 \mu \mathrm{m}, \varepsilon_{r}=2$ \\
Width of the structure $w$ & $1 \mathrm{~m}$ \\
Length of electrode $l$ & $0.05 \mathrm{~m}$ \\
Thickness of metal electrode and & $d_{\mathrm{m}}=10 \mu \mathrm{m}$ \\
freestanding layer & $20 \mu \mathrm{Cm}^{-2}$ \\
Tribo-charge surface density $\sigma$ & $1 \mathrm{~cm}$ \\
Electrode gap $g$ & $0 \mathrm{~mm}^{-1}$ \\
Freestanding height $h$ & $1 \mathrm{~ms}^{-1}$ \\
Motion average velocity $v$ &
\end{tabular}




\section{Graphical abstract}
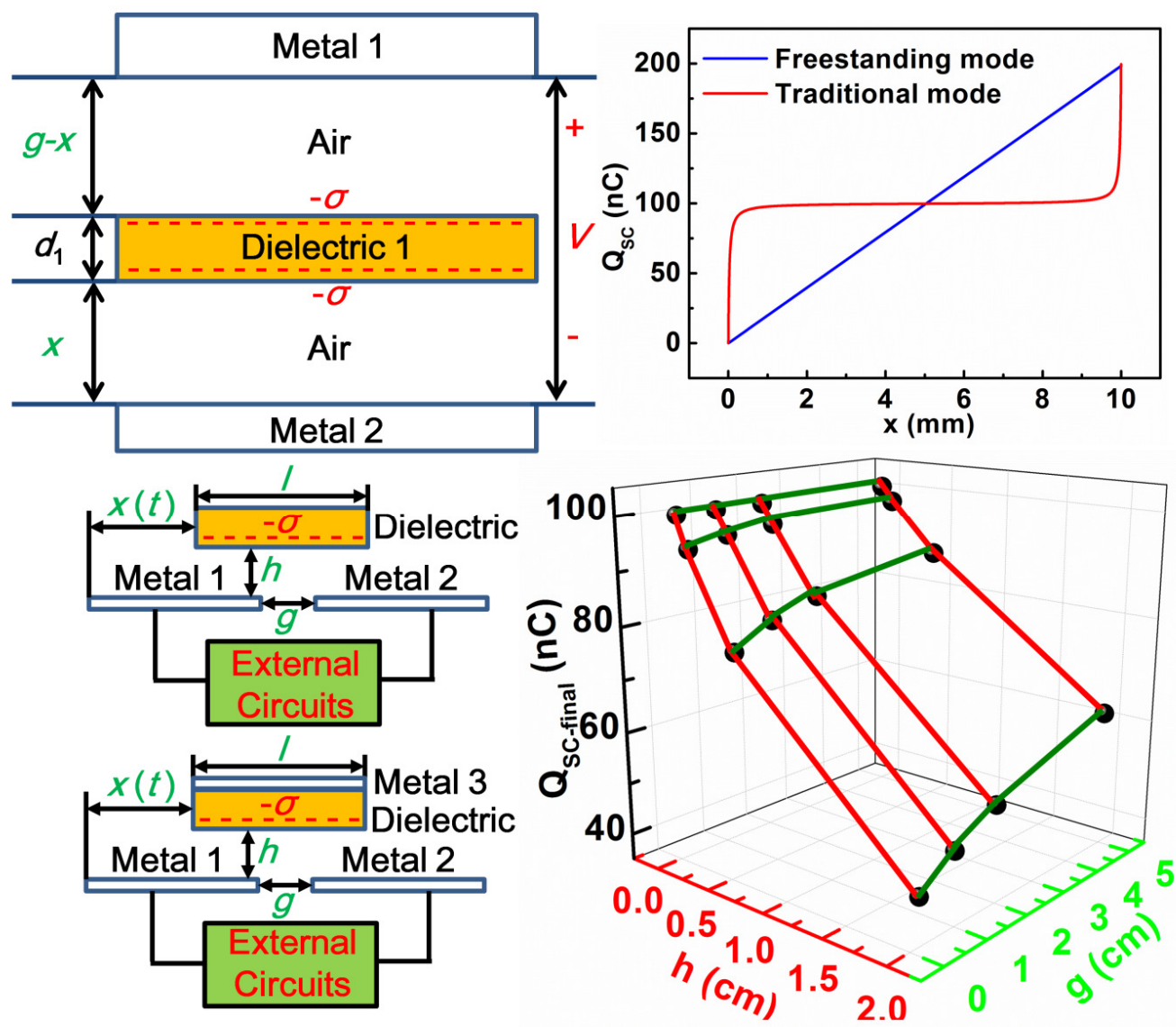